\title{
Acetylation of Asiatic Acid and Its Hepatotoxicity Prediction
}

\author{
Ida Musfiroh ${ }^{1,2^{*}}$, Ita Puspitasari ${ }^{2}$, Ahmad Muhtadi $^{2}$, Rahmana E. Kartasasmita $^{1}$, Slamet I. Surantaatmadja ${ }^{1}$ \\ ${ }^{1}$ School of Pharmacy, Bandung Institute of Technology, Jalan Ganesha 10, Bandung, 40132, Indonesia. \\ ${ }^{2}$ Faculty of Pharmacy, Universitas Padjadjaran, Bandung, Indonesia.
}

Received: 6 November 2014 / Accepted: 30 November 2014

\begin{abstract}
:
Asiatic acid (AA) is one of the triterpenoids isolated from pegagan (Centella asiatica (L.) Urb.), which has the antiinflammatory activity. The present studies were aimed to synthesize triacetyl asiatic acid (2,3,23-triacetooxy-12-ene asiatic acid) and to predict its hepatotoxicity properties. Acetylation was carried out using acetic acid anhydride and $\mathrm{ZnCl}_{2}$ as a catalyst, while the hepatotoxicity properties were studied using Accelrys Discovery Studio 2.5 software. The structure was elucidated with IR, Mass and NMR spectroscopic methods. The results showed that the product of synthesis was confirmed as triacetyl asiatic acid, and the yield was $31.79 \%$. The prediction of its hepatotoxicity revealed that triacetyl asiatic acid is less hepatotoxic than AA.
\end{abstract}

Key words: Acetylation, ADMET prediction, Asiatic acid

\section{Introduction}

Pegagan extract has three different triterpenoids, asiaticoside, asiatic acid, and madecassic acid. Among the three triterpenoids, asiatic acid is the most important compounds for biological activity. Structurally, asiatic acid has three functional groups, the hydroxy group at $\mathrm{C}(2), \mathrm{C}(3)$, and $\mathrm{C}(23)$, olefin group at $\mathrm{C}(12)$, and a carboxylic acid group at $\mathrm{C}(28)$ (Figure 1) [1].

Some study of structure-activity relationship previously showed that lipophylic transformation, such as the formation of acetate with three hydroxyl groups in the A ring of asiatic acid structure are importance to improve wound healing activity [2], hepaptoprotective effects, and the modification at three hydroxyl groups of asiatic acid has been reported using acetic anhydride and pyridine as a catalyst for 8 hours $[3,4]$. Previously, we published structure activity relationships that modification by adding acetate group to the hydroxyl group on the A ring showed higher affinity than asiatic acid. Likewise, ADMET properties of the compounds have been reported

*Corresponding author: Ida Musfiroh,

E-mail: idamusfiroh@unpad.ac.id using Preadmet software [5]. However, their hepatotoxicity prediction has not been reported yet. The use of in silico methods can reduce time and cost at a drug development stage. Predicting the ADME/Tox properties of molecules could prevent failure of the compounds targeted before reach the clinic phase [6].

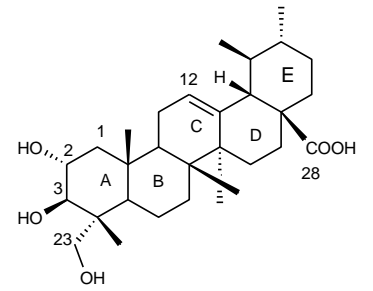

Figure 1. Structure of Asiatic Acid.

In this study, we synthesized triacetyl asiatic acid using acetic anhydride and $\mathrm{ZnCl}_{2}$ as a catalyst and to predict the hepatoxicity properties using Accelrys Discovery Studio 2.5 software.

\section{Computational Method}

\section{Materials}

The materials used in this experiment were Asiatic acid (Sigma Aldrich), acetic anhydride (Merck), aquadest, 
ethyl acetate (Bratachem), chloroform (Merck), methanol (Merck), and zinc chloride (Merck).

TLC GF254 (Merck), FTIR spectrophotometer (Shimadzu, IR Prestige-21), Mass Spectrometer (Waters), Nuclear. Magnetic Resonance (NMR) spectra were taken on a Agilent $500 \mathrm{MHz}$ for ${ }^{1} \mathrm{H}$ NMR, and tetramethylsilane (TMS) was used as an internal standard. Chemical shifts $(\delta)$ were recorded in ppm. ChemDraw Ultra v.8.0.3 software (Cambridge Soft Co.) 2003 version, Hyperchem 7 software, Accelrys Discovery Studio 2.5 software.

\section{Methods}

\section{Acetylation of Asiatic Acid}

$10 \mathrm{mg}$ of zinc chloride and acetic acid anhydride (0.472 $\mathrm{ml}, 0.35 \mathrm{mmol}$ ) were refluxed for 10 minutes at a temperature of $60-70{ }^{\circ} \mathrm{C}$ while stirring with a magnetic stirrer. The mixture was slowly added with asiatic acid (50 mg, $0.1 \mathrm{mmol}$ ), stirred with vigorous stirring, heated again for 1 hour, cooled until a white precipitate was formed. Furthere, the mixture was filtered and the precipitate washed with ice water until the sour smell disappeared. Purification was carried out by preparative thin layer chromatography using eluent chloroform: methanol $(9: 1, \mathrm{v} / \mathrm{v})$. The structure was elucidated with IR, Mass and NMR spectroscopic methods.

\section{Hepatotoxicity prediction}

The depiction of two dimensional structures used ChemDraw Ultra v.8.0.3 software (Cambridge Soft Co.), whereas geometry optimization used Hyperchem 8.07 software. Hepaptotoxicity prediction was carried out using ADMET Descriptor at Accelrys Discovery Studio 2.5. software [8] and hepatotoxicity descriptors were selected. (accelrys.discoverystudio.25.ADMET/ ADMET/admet_admet_descriptors).

\section{Results and Discussion}

\section{Chemistry}

Asiatic acid synthesis began by heating zinc chloride and acetic acid anhydride for 10 minutes. Acetic acid anhydride reagent acts as an acetyl donor. It is more reactive than the other carboxylic acids, so that the esterification reaction can more easily occur. The use of zinc chloride serves as a Lewis acid catalyst that can accelerate the esterification reaction. Zinc chloride is an electron acceptor, which will activate the acetyl group of acetic acid anhydride that can be attacked by nucleophilic, the hydroxyl group of the asiatic acid. The esterification reaction is reversible in the presence of water molecules so the reaction using acetic acid anhydride to avoid product of reaction hydrolyzed.

The TLC resulted that the $\mathrm{R}_{\mathrm{f}}$ of stain product was 0.88 , in comparison to AA with 0.48 . The larger value of $R_{f}$ was caused by substitution of a hydroxyl group with an acetyl group led to more polar compounds turn out to be nonpolar. The compound was obtained as white solid (31.79 $\%$ ). The IR absorptions indicated the presence of hydroxyl in carboxylic group (3477.66 $\left.\mathrm{cm}^{-1}\right)$ and ester carbonyl groups $\left(1745.11 \mathrm{~cm}^{-1}\right)$. The ESI MS-MS exhibited ion at $\mathrm{m} / \mathrm{z}=614.7616$ which was accordance with the formula $\mathrm{C}_{35} \mathrm{H}_{54} \mathrm{O}_{8}$. It was showed that asiatic acid was substituted with three of acetyl groups. The fragmentation was showed disconnection on the release of one of acetyl $\left(\mathrm{CH}_{3} \mathrm{CO}^{-}\right)$, two of acetyl, and three of acetyl, respectively. The resonance of ${ }^{1} \mathrm{H}-\mathrm{NMR}$ spectra $\left(500 \mathrm{MHz}, \mathrm{CDCl}_{3}\right) \delta 5.26(\mathrm{t}, 1 \mathrm{H}), 5.15-5.07(\mathrm{~m}, 1 \mathrm{H}), 5.0$ $(\mathrm{d}, 1 \mathrm{H}), 3.86(\mathrm{~d}, 1 \mathrm{H}), 3.58(\mathrm{~d}, 1 \mathrm{H}), 2.09,2.02,1.98,1.11$, $1.08,0.88,0.79(\mathrm{~s}, 3 \mathrm{H}), 0.94(\mathrm{~d}, 3 \mathrm{H}), 0.86(\mathrm{~d}, 3 \mathrm{H})$. The structure and spectrums were given in Figure 2 and 3. ${ }^{1} \mathrm{H}-\mathrm{NMR}$ spectroscopic data of the structure (Figure 3) were compared with the asiatic acid (Figure 4). It showed three of methyls at $\delta 2.09 ; 2.02 ; 1.98(\mathrm{~s}, 3 \mathrm{H})$. These facts in combination with IR absorptions for carbonyl of ester revealed that the compound had 126 mass units more higher than asiatic acid $\left(\mathrm{C}_{30} \mathrm{H}_{48} \mathrm{O}_{5}\right)$ and which was further confirmed by ${ }^{1} \mathrm{H}$ NMR. Finally, the product was concluded as 2,3,23-triacetooxy-12-ene asiatic acid or triacetyl asiatic acid.

Synthesis of triacetyl asiatic acid by acetylation on applying $\mathrm{ZnCl} 2$ as catalysts (Lewis acid) are considered 
more effective than using pyridine as a catalyst (alkaline) in terms of time [7].

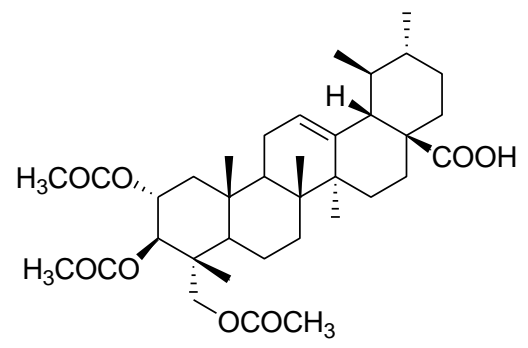

Figure 2. Structure of triacetyl asiatic acid.

Modification of C2, C3, C23 of asiatic acid was done by acetylation using pyridine taking much more time on 8 hours [3]. In addition, zinc chloride activating acetyl group in acetic acid anhydride acetylation process that can be occur at 1 hours of reaction. Acetylation method using acetic anhydride and $\mathrm{ZnCl}_{2}$ as a catalyst can be used as an alternative for synthesis of triacetyl asiatic acid.

\section{In Silico Study}

\section{Geometry optimization}

Modified two-dimensional structure of asiatic acid and triacetyl asiatic using ChemDraw Ultra v.8.0.3 software (Cambridge Soft Co.). Geometry optimization of asiatic acid and its modification using Hyperchem 8.07 software performed using the $\mathrm{MM}+$ method with Polak-Ribiere algorithm and convergence limit $0.1 \mathrm{kcal} / \AA \AA$ mol.

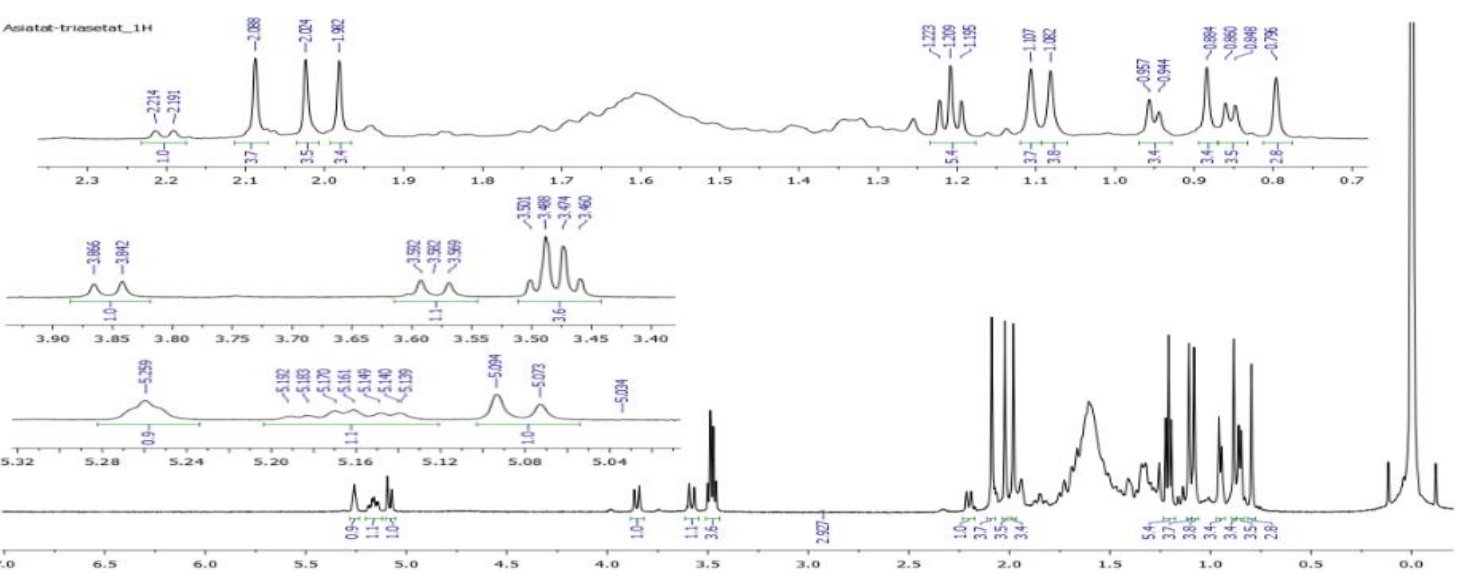

Figure 3. ${ }^{1} \mathrm{H}$ NMR spectrum of triacetyl asiatic acid.

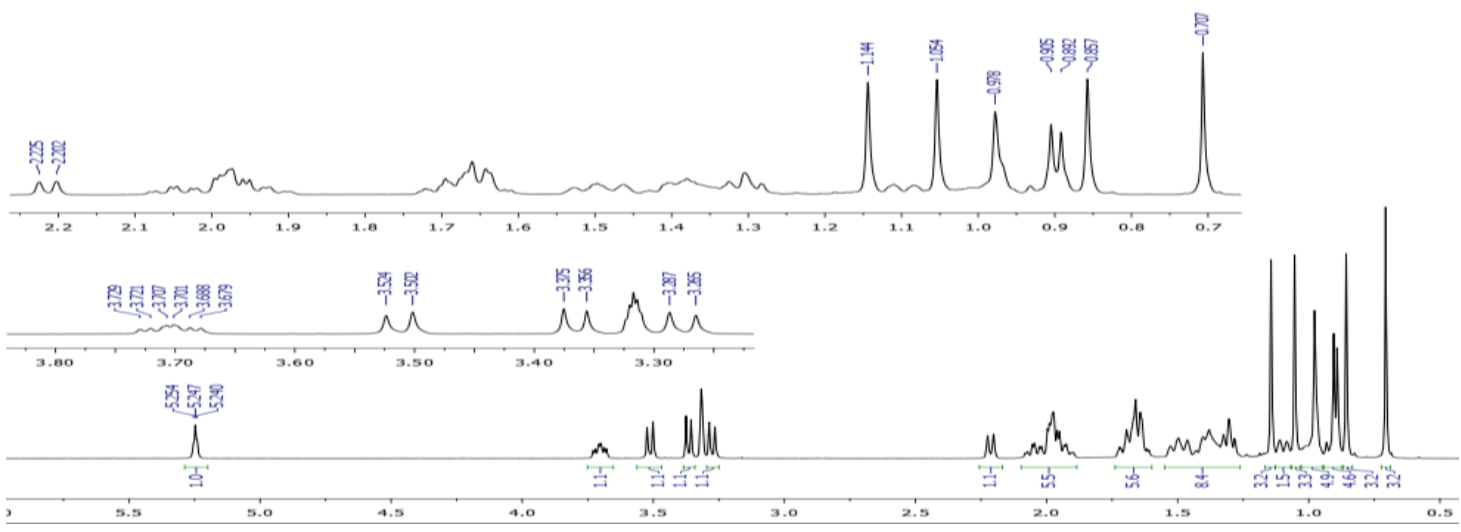

Figure 4. ${ }^{1} \mathrm{H}$ NMR Spectrum of asiatic acid. 
Table 1. ADMET Descriptor prediction of asiatic acid and its derivatives.

\begin{tabular}{lc}
\hline Compound & Hepatotoxicity \\
\hline Asiatic acid & 0.708 \\
Triacetyl asiatic acid & 0.496 \\
\hline
\end{tabular}

Hepatotoxicity: level $1=$ Toxic $(>0,5)($ Accelrys Discovery Studio Client 2.5)

Molecular mechanics method is a method of molecular modeling based on the laws of classical physics; it does not involve electronic transitions such as the semi-empirical, so it can be run with a low-speed computers or specification simple and very suitable for the analysis of molecules with thousands of atoms.

\section{Hepatotoxicity properties}

Hepatotoxicity prediction used to predict the occurrence hepatotoxicity of human dose-dependent. Asiatic acid (AA) has a value $>0.5$ indicates that the compound is likely to have hepatotoxic properties. Hepatotoxicity value of triacetyl asiatic acid is smaller than AA, so the possibility of toxic properties of compound is smaller than AA (Table 1).

\section{Conclusions}

Known compound was identified as a triacetyl asiatic acid (2,3,23-triacetooxy-12-ene asiatic acid). Its hepatotoxicity property prediction has less risk than AA.

\section{Acknowledgment}

This research was supported by Riset KK ITB 2014.

\section{References}

[1] B.S. Jeong, Structure-activity relationship study of asiatic acid derivatives for new wound healing agent, Arch. Pharm. Res., 29, 2006, 556-562.

[2] P.J. Shim, J.H. Park, M.S. Chang, M.J. Lim, D.H. Kim, Y.H. Jung, S.S. Jew, E.H. Park, and H.D. Kim, Asiaticoside mimetics as wound healing agent, Bioorg. Med. Chem. Left., 6, 1996, 2937-2940.

[3] L.X. Zhao, Hg. Park, Ss. Jew, M.K. Lee, Y.C. Kim, P. Thapa, R. Karki, Y. Jahng, B.S. Jeong, and E.S. Lee, Modification of C11, C28, C2,3,23 or C2, 23, 28 functional groups on asiatic acid and evaluation of hepatoprotective effects, Bull. Korean Chem. Soc., 28(6), 2007, 970-976.

[4] B.S. Jeong, C.K. Young, and S.L. Eung, Modification of C2,3,23,28 functional groups on Asiatic acid and evaluation of hepatoprotective effects, Korean Chem. Soc., 28(6), 2007, 977-982.

[5] R.E. Kartasasmita, I. Musfiroh, A. Muhtadi, and S. Ibrahim, Binding affinity of asiatic acid derivatives design against Inducible Nitric Oxide Synthase and ADMET Prediction, Journal of Applied Pharmaceutical Science, 4(02), 2014, 075-080.

[6] T.J. Carlson and M.D. Segall, Predictive, computational models of ADME properties, Curr. Drug Disc., 2002, 34-36.

[7] F.A. Carey and R.J. Sundberg, Advanced Organic Chemistry, Part B: Reaction and Synthesis, 4th 2001, Edition.New York: Kluwer Academic/Plenum Publishers.

[8] Accelrys, ADMET Descriptors in Discovery Studio, http://accelrys.com/products/datasheets/ds_admet_descriptors.p df, [Accessed at Sept, 2013]. 